\title{
THE DEVELOPMENT OF MECHATRONIC ACTIVE CONTROL SYSTEM OF TOOL SPATIAL POSITION IN PARALLEL KINEMATICS MACHINE TOOL
}

\author{
Vasil B. Strutinsky, Anatoliy S. Demyanenko \\ National Technical University of Ukraine "KPI", Faculty of Mechanical Engineering, Kyiv, Ukraine \\ e-mail:kvm_mmi@mail.ru; a.s.demyanenko@gmail.com
}

\begin{abstract}
The method and the mechatronic system of active control of the dynamic spatial positioning of the executive body of a parallel kinematics machine tool are proposed. By means of geometric modeling, the basic analytical relations that characterize the interconnection between the rods of the mechatronic control system and the power rods of the parallel kinematics machine tool are calculated. The analytical dependencies that characterize errors of the spatial position of the executive body of the parallel kinematics machine tool are set out. The prototype of the mechatronic system of active control of the dynamic spatial position of the executive body of the parallel kinematics machine tool has been manufactured and its presetting and viability checking made. The special equipment to control the exact position of the tool of the parallel kinematics machine tool when it comes to the position is proposed.
\end{abstract}

Keywords: parallel kinematics machine tool, accuracy, mechatronics, calibration

\section{Introduction}

Parallel kinematics machine tools are progressive manufacturing equipment. The main advantages of parallel kinematic machine tools are low material and energy intensity (Weck and Staimer, 2000). But as a result, there is a decrease in stiffness of the machine tool bearing system and, therefore, low accuracy and poor dynamic characteristics of engines (Strutinsky, 2012). Briot and Bonev (2007) stated theoretically that parallel robots are more accurate than serial ones, but in practice small errors in the drive system of 6 rods can cause the significant errors in the executive body location and its movement trajectory. Therefore, one of the efforts to increase the accuracy of parallel kinematics machine tools and robots can be their structural improvement. Nowadays, there are a significant number of investigations that are dedicated to the structural improvement of parallel kinematics machine tools (Dindorf and Laski, 2010; Guan, 2012; Huang, 2010).

Though there is a lot of designs of parallel kinematics machine tools and robots, the actual problem is still to achieve their high parameters of the kinematic and, especially, dynamic accuracy (Pandilov and Dukovski, 2012). The problem of the position accuracy of planar kinematically redundant parallel robots was considered in details in (Kotlarski et al., 2012). It was proposed to use the optimization of the redundant actuator position in a discrete manner. The authors used several exemplarily chosen trajectories on which they showed the improvement in terms of the accuracy of that planar kinematically redundant parallel mechanism. To some extent, those methods can be extrapolated to the spatial parallel kinematics mechanism.

On the other hand, the improved accuracy of spatial parallel kinematics machine tools is usually held by means of periodical calibration (Ibaraki et al., 2004; Joubair et al., 2014; Wu et al., 2014). The perspective concept of calibrating the parallel kinematics machine using an exteroceptive sensor-camera, which is measuring the end-effector position was discussed by Andreff and Martinet (2009). The methodology offered by the authors was based on using an inverted 
camera projection model which reduced the number of kinematic parameters to identify. There is another interesting research work (Szatmári, 2007), in which the author made the accuracy test of a hexapod-type machine tool by means of the laser interferometer which was used to take bidirectional repeated measurements along $X$ and $Y$ axes. The results showed that the accuracy of motion of the hexapod mechanism was rather poor, but the repeatability of the measurements was very accurate. So, as the errors have a systematic character, the promising way to improve the accuracy of parallel kinematics machine tools and robots is to correct these errors through the controller, on the CNC level.

All these developments do not provide full improvement of the accuracy of parallel kinematics machine tools especially during processing of a workpiece. But they show a promising direction to increase the accuracy of parallel kinematics machine tools, which is the introduction of a closed loop measuring system providing the possibility of correcting control signals for actuators and, accordingly, improving the accuracy of such machines during processing.

The main objective of this work is to develop a method to improve the accuracy of parallel kinematics machine tools through implementation of an active control system of the dynamic spatial position of its platform on which the tool is mounted. In order to achieve this goal, analytical dependencies that determine the regularity of work of the active control system of the dynamic spatial position of the tool will be discussed. The dependencies between lengths of the rods of the proposed measuring mechanism and the parallel kinematics machine tool rods as well as analytical dependencies relating small displacements of the executive body and lengths of the rods will be set. The prototype of the proposed system is described. It is shown that an additional calibration error in the output tool position of the executive body of the parallel kinematics machine tool should be taken into account. Therefore, the construction of special equipment to track these errors periodically is offered.

\section{The method and the mechatronic system for active control of dynamic spatial positioning of the tool in a parallel kinematics machine tool}

The parallel kinematics machine tool is based on the mechanism of a hexapod type and has six rods of variable lengths $L_{1}, \ldots, L_{6}$, which are connected with the mobile executive body PL, where tool $I$ which is designed to handle the contoured surface of the workpiece $D$ is mounted (Fig. 1).

The machine tool has a base with two power belts $\mathrm{H} 1$ and $\mathrm{H} 2$. The hinged support rods of variable length are mounted on this base.

The tool moves along a complex trajectory, thus changing its transverse angular position in the process of machining of the workpiece. Changing the spatial position of the executive body is achieved by changing the length of the rods of the machine tool. The terms of the executive body in space are determined by its translational displacement of some point (pole) and transverse angular position of the executive body relative to the pole.

Assume some center point $p$ of the tool as a pole. Therefore, the law of the tool movement will be described by the trajectory of the pole and current Euler angles that define the angular position of the tool at each point of the trajectory. The Euler-Krylov angles $\psi, \theta, \varphi$ which define the rotation angles of the executive body relative to axes $O x, O y$ and $O z$ are assumed (see Fig. 1). The position of the tool will be defined as a vector which has six components. Three of them are linear coordinates (set of linear movements $x, y, z$ ) of the tool, and three rotational (angular values $\psi, \theta, \varphi$ ). Accordingly, the position of each point $U$ of the executive body is characterized by the relevant vector which has been defined in space of 6 dimensions.

Using the injected vector has a certain inconvenience caused by heterogeneity of its components. Therefore, it is proposed to use a modified vector, which differs by angular values brought 


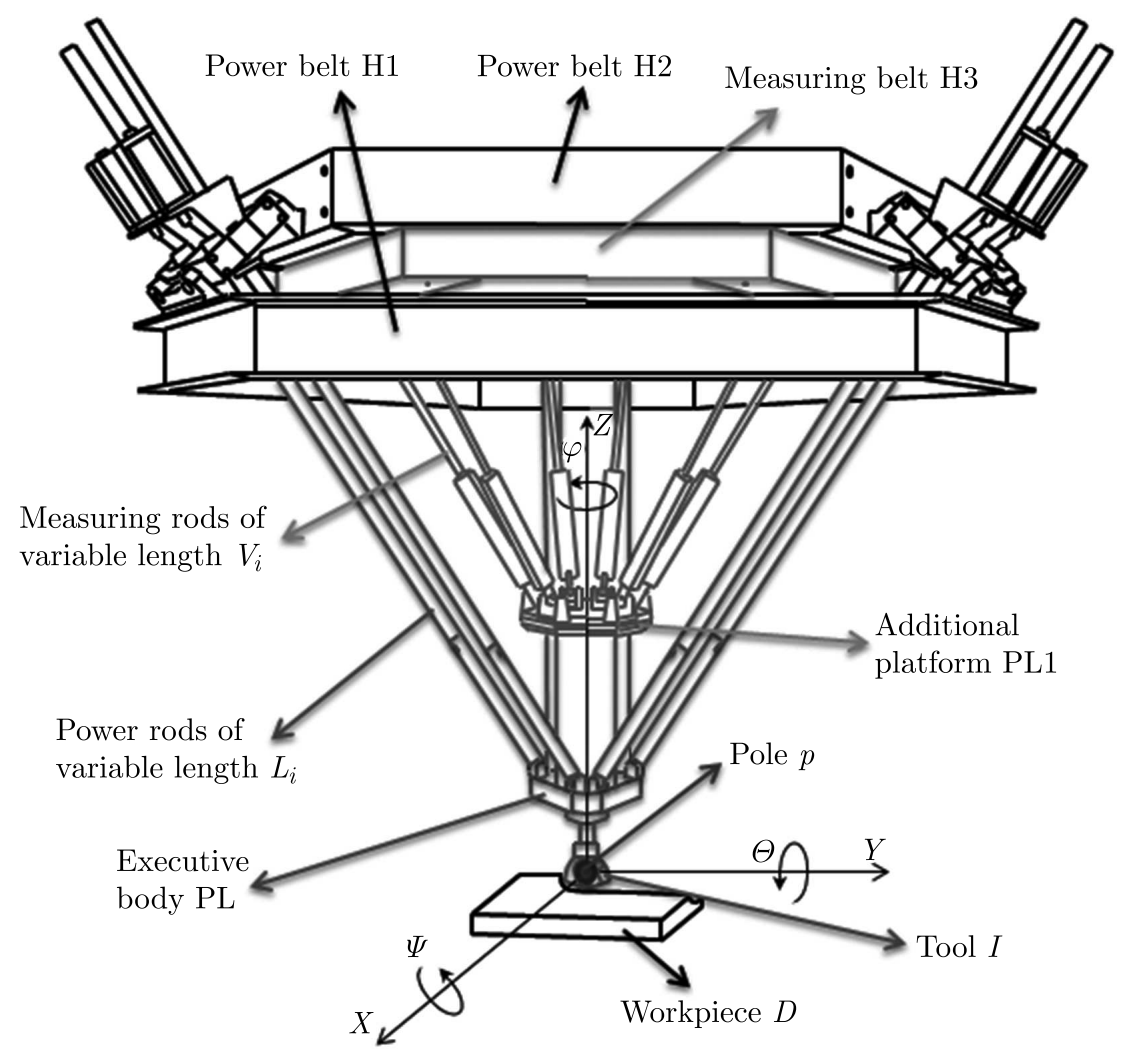

Fig. 1. The scheme of the parallel kinematics machine tool with a mechatronic system of active control of dynamic spatial positioning of the tool

in line. Taking this into count, the parameter vector that defines the position of the point $U$ can be written as

$$
\mathbf{X}_{U}=\left[x, y, z, m_{\psi} \psi, m_{\theta} \theta, m_{\varphi} \varphi\right]^{\mathrm{T}}
$$

where $m_{\psi}, m_{\theta}, m_{\varphi}$ are scale factors with the coordinates dimension.

In general, the vector that defines the position of the point $U$, can be written as

$$
\mathbf{X}=\left[x_{i}\right] \quad i=1,2, \ldots, 6
$$

where $x_{i}$ is the corresponding component of the vector.

In the process of programming of the law of motion for a parallel kinematics machine tool, the changes in the time $t$ component of the vector $\mathbf{X}$ are set as

$$
\mathbf{X}_{1}=\left[x_{i}(t)\right]
$$

As a result of solution of the inverse problem, the kinematics vector of $l$-coordinates is calculated.

The resulting vector of $l$-coordinates is presented in the form

$$
\mathbf{L}=\left[l_{j}\right] \quad j=1,2, \ldots, 6
$$

The vector components given as functions of time are

$$
l_{i}=l_{i}(t)
$$

Necessary laws for $l$-coordinates changes are implemented by the drives. As a result of changes of lengths of the rods, the executive body of the machine tool is positioned in the appropriate position, which is characterized by the vector $\mathbf{X}$. 
The accuracy of the executive body setting in the required position is determined by the vector of errors

$$
\boldsymbol{\delta} x_{i}=\mathbf{X}_{0}-\mathbf{X}_{U}
$$

where $\mathbf{X}_{0}$ is a vector describing the desired position that has been set in the CNC system and $\mathbf{X}_{U}$ is the vector describing the actual position of the executive body of the parallel kinematics machine tool.

The vector $\boldsymbol{\delta} x_{i}$ depends on numerous random factors. Defining of the vector of errors as a function of time is a task of the mechatronic system of active control of dynamic spatial positioning of the executive body of the parallel kinematics machine tool.

The developed method and the mechatronic system of active control of dynamic spatial positioning of the tool is based on making use of an additional parallel kinematics mechanism which has six measuring rods of variable length $V_{1}, \ldots, V_{6}$, connecting with the executive body with power belt $\mathrm{H} 3$ which is rigidly connected with power belts $\mathrm{H} 1$ and $\mathrm{H} 2$. The measuring rods have sensors that record changes in lengths of the rods. If one changes the $x$-coordinate according to relationship (2.3), the length of the rods is changing and, therefore, the vector

$$
\mathbf{V}=\left[v_{j}\right] \quad j=1,2, \ldots, 6
$$

According to these values, the executive body positioning error is defined by formula (2.6).

\section{Basic analytical dependencies characterizing the consistent pattern of the active control system}

The $l$-coordinates vector is functionally dependent on the vector of input parameters $\mathbf{X}_{i}$. This dependence is nonlinear and, in general, can be written as

$$
\mathbf{L}=\mathbf{F}(\mathbf{X})
$$

where $\mathbf{F}(\mathbf{X})$ is a vector whose components in general can describe the dependences for the $l$-coordinates of the components of the vector of input parameters and time

$$
\mathbf{F}(\mathbf{x})=\left[l_{j}\left(\left[x_{i}\right], t\right)\right] \quad j=1,2, \ldots, 6
$$

$\mathbf{V}$ is the vector of coordinates and also depends on the vector of input parameters $x_{i}$ according to the equation

$$
\mathbf{V}=\mathbf{F}_{1}(\mathbf{X})
$$

where $\mathbf{F}_{1}(\mathbf{x})=\left[v_{j}\left(\left[x_{i}\right], t\right)\right], j=1,2, \ldots, 6$.

By combining dependencies (3.2) and (3.3), we can get a relationship between the $l$-coordinates and $v$-coordinates of our machine tool in the form

$$
\mathbf{V}=\boldsymbol{\Phi}(\mathbf{L})
$$

Dependency (3.4) can be applied only to a specific law of the executive body movement set in form (2.4).

To study the patterns of communication of the $v$-coordinates and $l$-coordinates, the inherent law of the executive body movement corresponding to the processing of a convex surface of an ellipsoid type is chosen (Fig. 2).

The tool moves along a curved trajectory when processing the convex surface (Fig. 3). Let us assume the shape of the selected area as a trajectory of an arc of a circle with radius 


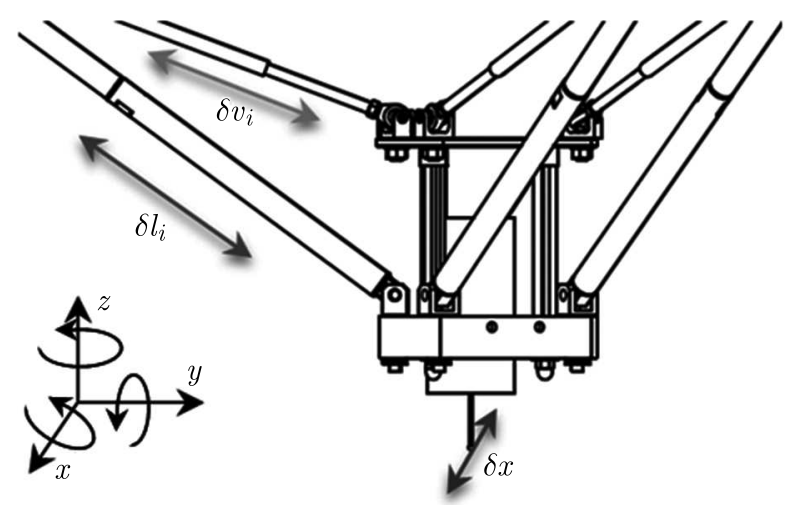

Fig. 2. Relationship between infinitesimal changes of $x$-coordinate, $l$ - and $v$-coordinates

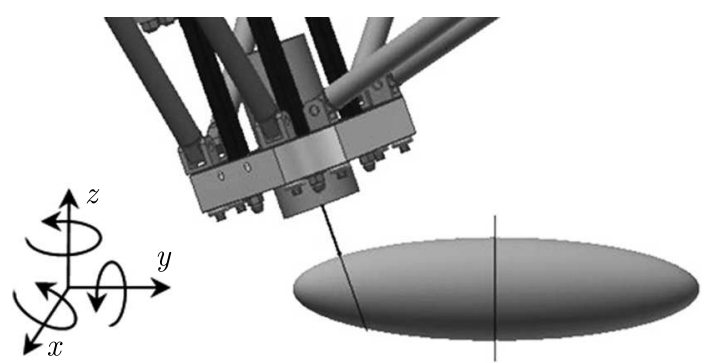

Fig. 3. The scheme of configuration changes of the parallel kinematics machine tool when processing a convex surface of an ellipsoid type

$R(x=0, \theta=0, \varphi=0)$. By means of geometric modeling in Autodesk Inventor, length of each rod of the machine tool for the fixed values of the rotation angle $\psi$ is defined

$$
\begin{aligned}
\boldsymbol{\psi}=\left[\begin{array}{c}
-20 \\
-15 \\
-10 \\
-5 \\
0 \\
5 \\
10 \\
15 \\
20
\end{array}\right] \quad \mathbf{L}_{1}=\left[\begin{array}{l}
1159.303 \\
1144.890 \\
1132.842 \\
1123.325 \\
1116.476 \\
1112.392 \\
1111.135 \\
1112.724 \\
1117.134
\end{array}\right] \quad \mathbf{V}_{1}=\left[\begin{array}{l}
790.070 \\
746.319 \\
707.831 \\
675.820 \\
649.946 \\
635.945 \\
629.925 \\
633.758 \\
647.239
\end{array}\right] \ldots \\
\mathbf{L}_{6}=\left[\begin{array}{c}
1175.619 \\
1157.885 \\
1141.988 \\
1128.125 \\
1116.476 \\
1107.196 \\
1100.416 \\
1096.231 \\
1094.703
\end{array}\right] \quad\left[\begin{array}{l}
784.250 \\
742.082 \\
705.212 \\
674.808 \\
649.946 \\
637.841 \\
632.962 \\
637.633 \\
651.613
\end{array}\right]
\end{aligned}
$$

The resulting point values of the rods lengths are smoothed by cubic splines (Fig. 4).

Analyzing the graphs, we can see that the laws for the rods lengths of the machine tool and for the measuring rods are similar.

During the researches, the correlation between the power rods length of the machine tool and the measuring rods of the additional mechatronic mechanism was defined (Fig. 5). This relation- 


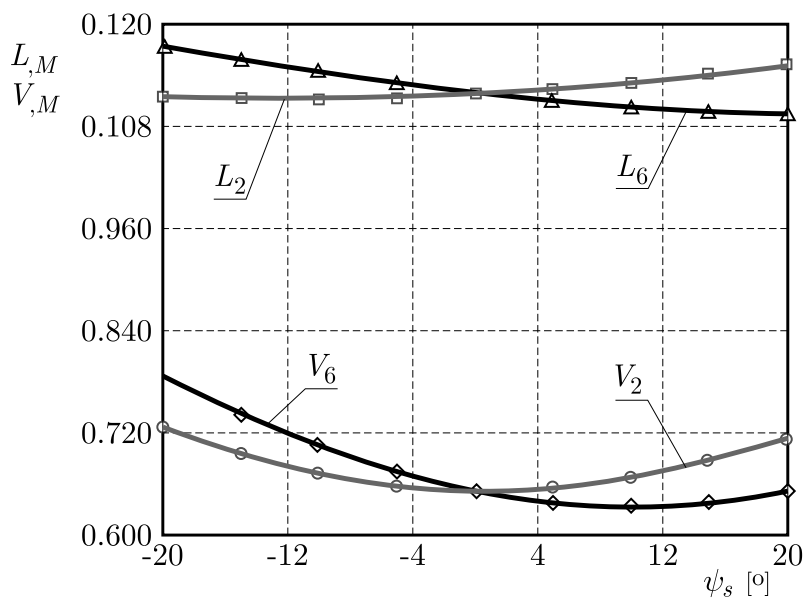

Fig. 4. The dependence between length of the rod and the angular distance while moving the tool along a convex surface such as an ellipsoid with radius of curvature $R=200 \mathrm{~mm}$

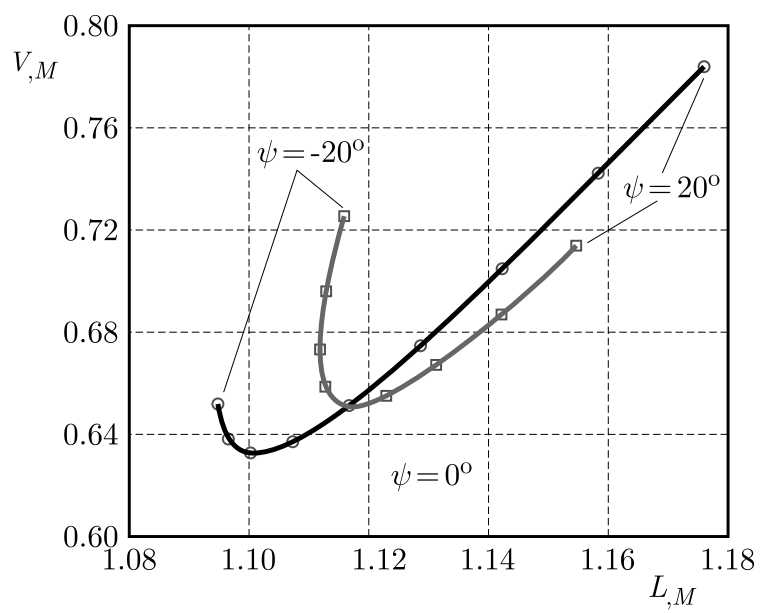

Fig. 5. The relationship between length of the rods of an additional mechanism and length ov the rods of the parallel kinematics machine tool

ship can be described by ambiguous dependencies with extremes. The presence of complex and ambiguous relationships of parameters requires a specific approach to determine the functional dependence for $v$-coordinates of $l$-coordinates. It is proposed to find these dependencies based on small increments of coordinates as shown below.

Let us define the relationship between infinitesimal changes of $x$-coordinates and $l$-coordinates. Each component of the vector $\mathbf{L}$ is a coordinate of a function type of 6 variables which are the $x$-coordinates. Using differentiation functions of several variables, we can find the vector of differentials of the $l$-coordinates

$$
\left[d l_{j}\right]=\sum_{i=1}^{6} \frac{\partial l_{j}}{\partial x_{i}} d x_{i} \quad j=1,2, \ldots, 6
$$

where $d l_{j}$ is a differential of the $j$-th $l$-coordinate.

All values that are dependent allow direct calculation. As a result, the partial derivatives $\partial l_{j} / \partial x_{i}$ are found.

Now we can write down equation (3.5) in a matrix-vector form

$$
d \mathbf{L}=\mathbf{M} d \mathbf{X}
$$


where the matrix $\mathbf{M}$ has relevant partial derivatives of the $l$-coordinate of the $x$-coordinates

$$
\mathbf{M}=\left[\begin{array}{ccc}
m_{11} & \cdots & m_{16} \\
m_{21} & \cdots & m_{26} \\
\vdots & \vdots & \vdots \\
m_{61} & \cdots & m_{66}
\end{array}\right] \quad m_{j i}=\frac{\partial l_{j}}{\partial x_{i}}
$$

If during solving all the input parameters are specified as functions of time $t$, then the components of the matrix can be found as differentials of a complex function

$$
m_{j i}=\frac{\partial l_{j}}{\partial t} \frac{\partial t}{\partial x_{i}}=\frac{\partial l_{j}}{\partial t} \backslash \frac{\partial x_{i}}{\partial t}
$$

During computing, it is possible that singularities may occur due to the advent of the component matrix $m_{j i}=0$ or $m_{j i}=\infty$. One reason for this phenomenon may be the condition

$$
\frac{\partial x_{i}}{\partial t}=\left\{\begin{array}{ll}
0 & \vee
\end{array} \quad \frac{\partial l_{i}}{\partial t}=\left\{\begin{array}{l}
0 \\
\infty
\end{array}\right.\right.
$$

These particular cases should be analyzed in the solution process of the direct kinematics problem.

Similarly, the dependence of the $v$-coordinates changes in the $x$-coordinate is determined

$$
d \mathbf{V}=\mathbf{Q} d \mathbf{X}
$$

where the matrix $\mathbf{Q}$ has components of relevant partial derivatives of the $v$-coordinates for the $x$-coordinates

$$
\mathbf{Q}=\left[\begin{array}{ccc}
q_{11} & \cdots & q_{16} \\
q_{21} & \cdots & q_{26} \\
\vdots & \vdots & \vdots \\
q_{61} & \cdots & q_{66}
\end{array}\right] \quad q_{j i}=\frac{\partial v_{j}}{\partial x_{i}}
$$

During the first phase of research, we confine ourselves to the case of the absence of infinite values of the matrices $\mathbf{M}$ and $\mathbf{Q}$.

For the average position of the executive body $\left(\psi=0^{\circ}\right)$, the components of matrix $\mathbf{M}$ and $\mathbf{Q}$ are calculated. For example, for the matrix $\mathbf{M}$ at this point, we have

$$
\mathbf{M}=\left[\begin{array}{cccccc}
-3.761 & -2.096 & 24.750 & 4.889 & 2.849 & 1.941 \\
-1.584 & -0.883 & 10.424 & 2.059 & 1.200 & 0.818 \\
1.375 & 0.766 & -9.047 & -1.787 & -1.041 & -0.710 \\
1.877 & 1.046 & -12.355 & -2.441 & -1.422 & -0.969 \\
1.957 & 1.090 & -12.880 & -2.544 & -1.482 & -1.010 \\
-2.369 & -1.320 & 15.591 & 3.080 & 1.795 & 1.223
\end{array}\right]
$$

Resulting matrix (3.12) is singular. Its determinant is zero and rank is one. This also applies to the matrix $\mathbf{Q}$.

Let us solve the equation that relates the differential of the $x$-coordinates and the $l$-coordinates. To do this, we define the differential of each $x$-coordinate as a function of 6 variables of the $l$-coordinates

$$
\left[d x_{i}\right]=\sum_{j=1}^{6} \frac{\partial x_{i}}{\partial l_{j}} d l_{j}
$$


Note that

$$
\frac{\partial x_{i}}{\partial l_{j}}=\frac{\partial x_{i}}{\partial t} \backslash \frac{\partial l_{j}}{\partial t}=\frac{1}{\frac{\partial l_{j}}{\partial t} \backslash \frac{\partial x_{i}}{\partial t}}=\frac{1}{m_{j i}}=\frac{\partial l_{j}}{\partial t} \frac{\partial t}{\partial x_{i}}=\frac{\partial l_{j}}{\partial t} \backslash \frac{\partial x_{i}}{\partial t}
$$

Hence, the ratios are defined by means of components of the matrix $\mathbf{M}$, and it is an inverse to them. Herewith

$$
d \mathbf{X}=\mathbf{N} d \mathbf{L}
$$

where $\mathbf{N}$ is a transpose matrix whose components are the components of the inverse matrix $\mathbf{M}$. Thus the differential of the $x$-coordinates can be expressed by differentials of the $l$-coordinates by the formulas

$$
d x_{i}=\sum_{j=1}^{6} n_{j i} d l_{j}
$$

where $n_{j i}=1 / m_{j i}$.

Similarly, a link of the $x$-coordinates and the $v$-coordinates can be found out

$$
d \mathbf{X}=\mathbf{P} d \mathbf{V}
$$

In a index form

$$
d x_{i}=\sum_{j=1}^{6} p_{j i} d v_{j}
$$

where the components of the matrix $\mathbf{P}$ are defined as $p_{j i}=1 / g_{j i}$.

Combining matrix vector eautions (3.15) and (3.17), we can define

$$
\mathbf{N} d \mathbf{L}=\mathbf{P} d \mathbf{V}
$$

In the index form

$$
\sum_{j=1}^{6} n_{j i} d l_{j}=\sum_{j=1}^{6} p_{j i} d v_{j}
$$

Formula (3.20) establishes a synonymous dependence of the $l$-coordinate and the $v$-coordinates.

Moving from the differentials $d x_{i}$ and $d l_{j}$ to the end increments of the corresponding values, we get

$$
\delta x_{i}^{l}=\sum_{j=1}^{6} n_{j i} \delta l_{j}
$$

The elements of $\mathbf{P}$ and $\mathbf{N}$ matrix establish a connection of two groups of changes of the physical coordinates. They allow us to establish a relationship for coordinate changes.

Equation (3.21) determines changes in the spatial position of the machine tool according to the $l$-coordinates changes.

Similarly, the $x$-coordinate by changes in the $v$-coordinates can be determined

$$
d x_{i}^{v}=\sum_{j=1}^{6} p_{j i} d v_{j}
$$


Equation (3.22) establishes the repositioning changes of the tool after measuring the $v$-coordinates. The spatial position errors of the tool can be find as the difference of the vectors

$$
\left[\Delta_{i}\right]=\left[\delta x_{i}^{l}\right]-\left[\delta x_{i}^{v}\right]
$$

The errors are calculated in a fixed position around the executive body, which is defined by the vector $\mathbf{X}_{0}$ and its corresponding vectors $\mathbf{L}_{0}$ and $\mathbf{V}_{0}$.

The vectors $\mathbf{L}_{0}$ and $\mathbf{V}_{0}$ depend on the $x$-coordinate and are set by formulas (3.1) and (3.4). Thus, there is an error in the output tool position which is defined by formula (2.6). To improve the accuracy of the calculation of changes in the spatial position of the tool by formula (3.33), it is proposed to conduct periodic adjustment of this dependency by experimental measurements of the exact position of the tool. To do this, the measurements of the exact position of the tool are done in a number of points $k=1,2, \ldots, K$. The actual position errors of the tool are identified by formula (2.6). The resulting array of errors

$$
\left[\delta x_{i}^{k}\right] \quad i=1,2, \ldots, 6 \quad k=1,2, \ldots, K
$$

is smoothed within the workspace which enables obtaining continuous values of the vector of errors $\left[\delta x_{i}^{k}\right]$. The actual spatial position errors are modified by equation (3.33) and are

$$
\left[\Delta_{i}\right]=\left[\delta x_{i}^{l}\right]-\left[\delta x_{i}^{v}\right]-\left[\delta x_{i}^{c}\right]
$$

\section{Structural implementation of the mechatronic active control system}

The improved accuracy and stability of the parallel kinematics machine is achieved by correction of control laws, which is realized directly in the CNC system. To achieve this goal, the measurements of the actual spatial position of the tool is made. The measurements are made by the mechatronic system of active control of dynamic spatial positioning of the tool. Its constructive implementation corresponds to the scheme shown in Fig. 1.

The system of active control has been implemented as a prototype. The executive body of the machine $\mathrm{P}$ moves in space by six rods of variable length $\mathrm{Li}$ and six measuring rods Vi (Fig. 6).

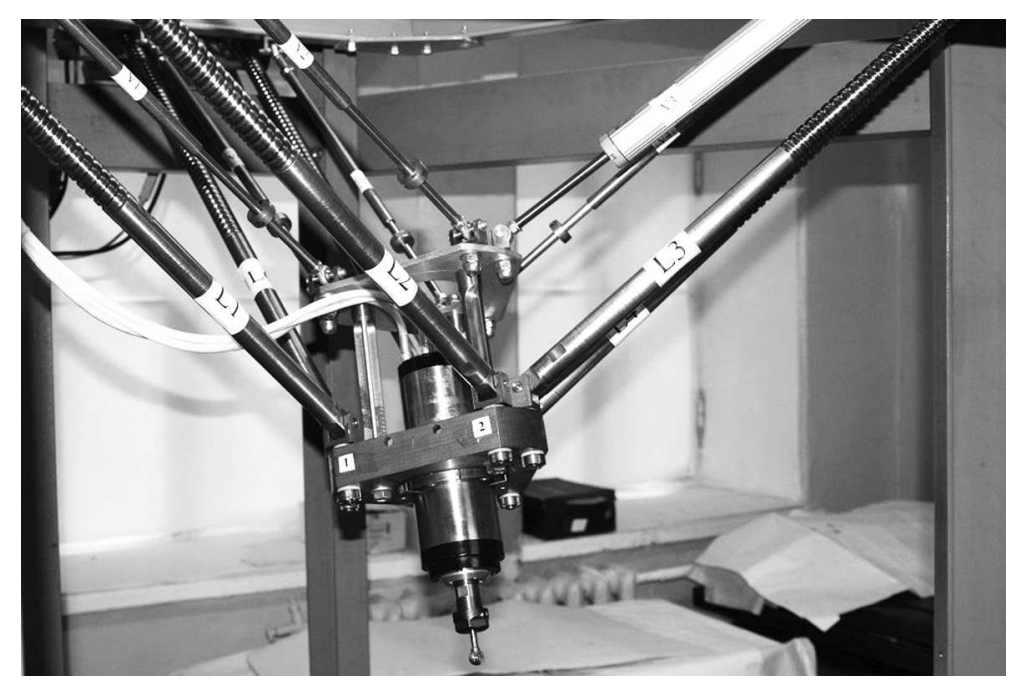

Fig. 6. Structural implementation of the mechatronic system of active control of dynamic spatial positioning of the tool of the parallel kinematics machine

To implement the active control of spatial positioning of the tool, an additional mechatronic mechanism with six measuring rods $\mathrm{V}$ has been made. The basis of the measuring rod is linear 
potentiometric displacement sensor PC-M-200. The voltage obtained from the sensor are handled by the analog-to-digital converter (ADC) M-DAQ14 which sends the obtained data to a personal computer for further analysis and processing. To convert an analog signal into a discrete one and display it on a virtual oscilloscope in LabView environment has been developed. Block Diagram and the User Interface of developed VI are shown in Figs. 7a and 7b, respectively.

(a)

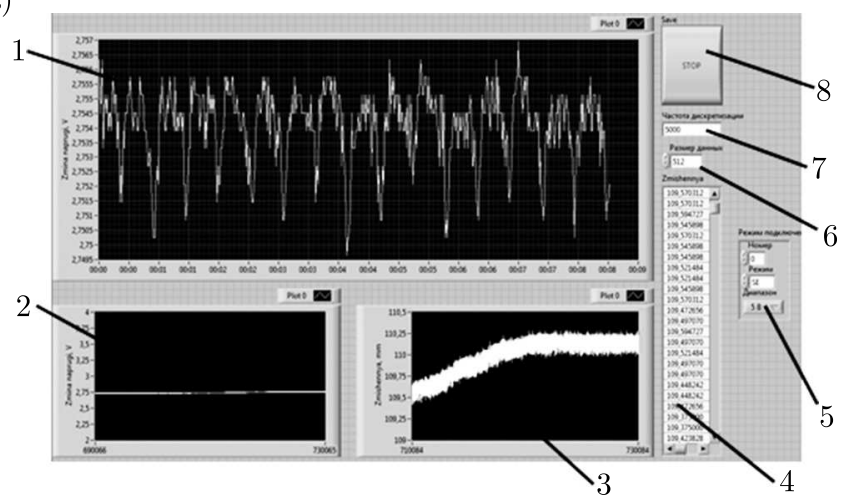

(b)

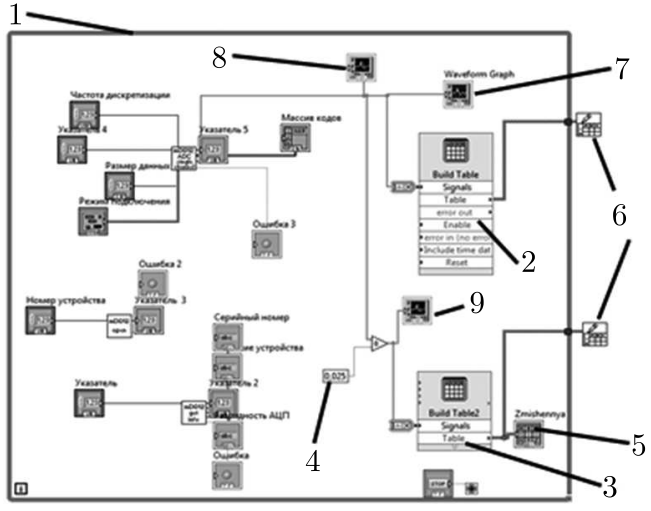

Fig. 7. The developed LabView VI: (a) user interface; (b) Block Diagram to display and record the data from ADC during measurements with the linear displacement sensor

In Waveform Graph 1 (Fig. 7a) voltage changes in the received signal at the time interval $0.08 \mathrm{~s}$ is displayed. Based on these values, we can control the level of noise and the accuracy of the measurements. The boxes of Waveform Chart 2, 3 present the voltage value received from the sensor and the linear displacement throughout the spectrum of measurements, respectively. Element 4 displays numerical values of the elongation of the sensor. In box 5 , we can adjust the measuring range: $\pm 1.25 \mathrm{~V}, \pm 2.5 \mathrm{~V}, \pm 5 \mathrm{~V}$ and $\pm 10 \mathrm{~V}$ and set the mode for measurement and ADC channels from which the voltage value is received. The range of the data processed by ADC is set box 6 . The frequency of analog signal 7 can be set in the range up to $20000 \mathrm{~Hz}$. Pressing «Stop» 8 completes the program display and record the data in the .xls or .txt format.

The cycle «While-Loop» 1 which provides the collection of data from the converter until «Stop» is clicked in the block diagram (Fig. 7b). Functions «Build table» 2, 3 form a table of values of the amplitude based on the received signal. Two tables are formed as a result: the input voltage $\mathrm{V}$ and the converted to millimeters data by means of using the pre-calculated numerical constants 4 . The value of elongation of the sensor rod is given in element 5 . To record the data, routines Write to Spreadsheet File.vi 6 is used. The real time display of the signal from the sensor is implemented in Waveform Graph, Windows 7, that provides visual control of the signal noise. The Waveform Chart 8 and Waveform Chart 9, display the voltage value and the corresponding elongation of the sensor rod.

The periodical calibration of the system to control the exact position of the executive body of the machine when its output is clearly fixed in the position within the workspace.

To set a fixed position, special devices (gauges) have been developed. These devices are a system of spheres that are located in well-defined positions within the workspace of the machine tool. The spheres are made of ceramic which has minimal thermal deformations. The spheres are placed in the holes of similar modules at the vertices of squares of the parts $100 \pm 0.002 \mathrm{~mm}$ (Fig. 8)

By means of these modules we can form flat or spatial structures of different configurations. Each module consists of base 1 in which precision spheres 2 with a diameter $35 \pm 0.001 \mathrm{~mm}$ are set. These spheres are fixed in the base with clamps 3. To determine the precision parameters of the machine tool with rectilinear motion and grooves 4 in the base are provided. 
(a)

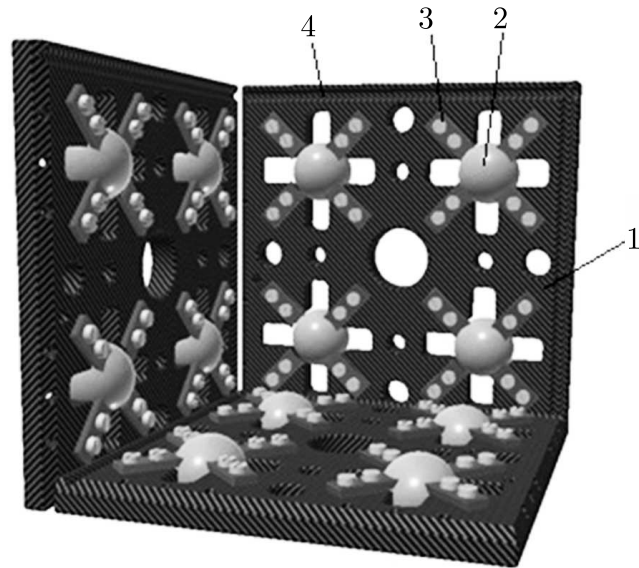

(b)

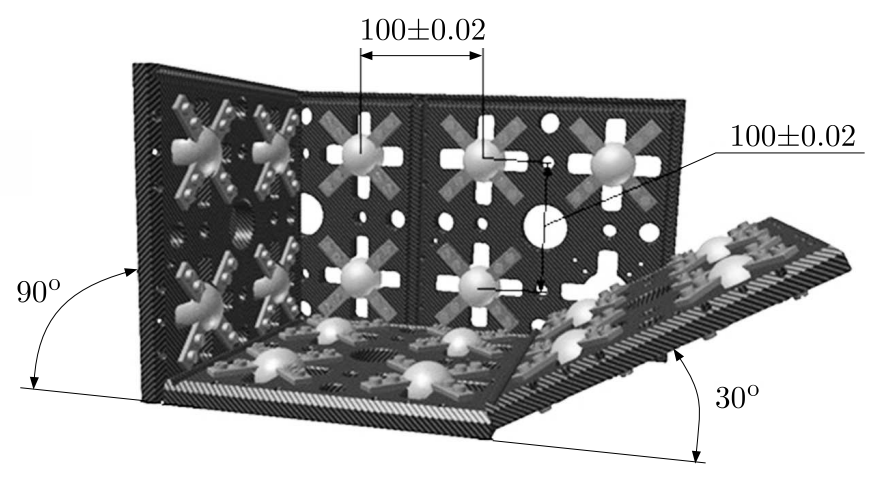

Fig. 8. Special equipment which consists of modules of the same type to determine the accuracy of the executive body output in positions: (a) disposition of modules in the cube form, (b) spatial disposition of modules

The developed equipment is set on the table of the parallel kinematics machine tool. To perform the calibration operation, the meter with a contact probe is set in the spindle. During the calibration, the output position of the executive body of the machine tool is determined which corresponds to spatial arrangement of the spheres of the developed equipment in the appropriate configuration.

\section{Conclusions}

In this paper, the method and the mechatronic system of active control of the dynamic spatial positioning of the executive body of the parallel kinematics machine tool which is implemented as an additional parallel kinematic mechanism with six measuring rods. The basic analytical relationships that determine the regularity of work of the active control system of the dynamic spatial positioning of the tool are proposed and additionally equated by means of geometric modeling. It is shown that the laws for the machine tool and the additional measuring mechanism yield similar changes of rods lengths. The proposed analytical method of connecting small movements of the executive body and changes in the rods length give us the opportunity to determine analytical formulas that characterize the spatial position error of the executive body of the parallel kinematics machine tool when the output is in a fixed position.

The structural implementation of the mechatronic system of active control of the dynamic spatial positioning of the executive body of the parallel kinematics machine tool is proposed and manufactured as a prototype. Its preliminary setup and test efficiency are held. It is noted that the resulting array of spatial position errors that can be found on the base of the proposed analytical equations contains the additional error in the output tool position which should be taken into account. It can be done by means of conducting periodic adjustment of theses formulas by experimental measurements of the exact position of the tool. Thereby, the construction of special equipment to determine the exact position of the executive body of the parallel kinematics machine tool and its calibration is proposed.

\section{References}

1. Andreff N., Martinet P., 2009, Vision-based self-calibration and control of parallel kinematic mechanisms without proprioceptive sensing, Intelligent Service Robotics, 2, 71-80

2. Briot S., Bonev I.A., 2007, Are parallel robots more accurate than serial robots, Transactions of the Canadian Society for Mechanical Engineering, 31, 445-455 
3. Dindorf R., Laski P., 2010, Design and experimental test of a pneumatic parallel manipulator tripod type 3UPRR, Acta Mechanica et Automatica, 4, 9-13

4. Guan L., 2012, Design, Analysis, and Prototyping of A 3xPPRS Parallel Kinematic Mechanism for Meso-Milling, Department of Mechanical and Industrial Engineering University of Toronto

5. HuAng H., 2010, A 6-DOF adaptive parallel manipulator with large tilting capacity, Robotics and Computer-Integrated Manufacturing, 28, 275-283

6. Ibaraki S., Yokawa T., Yoshiaki K., Masao N., 2004, A study on the improvement of motion accuracy of hexapod-type parallel mechanism machine tools (2nd Report) - A calibration method to evaluate positioning errors on the global coordinate system, Journal of the Japan Society for Precision Engineering, 40, 557-561

7. Joubair A., Slamani M., Bonev I., 2014, Kinematic calibration of a five-bar planar parallel robot using all working modes, Robotics and Computer-Integrated Manufacturing, 29, 15-25

8. Kotlarski J., Heimann B., Ortmaier T., 2012, Improving the pose accuracy of planar parallel robots using mechanisms of variable geometry, Advances in Robot Manipulators, 381-400

9. Pandilov Z., Dukovski V., 2012, Parallel kinematics machine tools: overview - from history to the future, Annals of Faculty Engineering Hunedoara, International Journal of Engineering, 10, $111-124$

10. Strutinsky S.V., 2012, Skhemna realizatsiya prostorovoyi systemy pryvodiv dlya manipulyuvannya obyektamy mashynobuduvannya (in Ukrainian), Naukovyy Zhurnal "Tekhnolohichni kompleksy", 2, 97-103

11. Szatmári S., 2007, Kinematic calibration of parallel kinematic machines on the example of the hexapod of simple design, Dissertation zur Erlangung des akademischen Grades Doktoringenieur, $146 \mathrm{p}$.

12. Weck M., Staimer D., 2000, On the accuracy of parallel machine tools: design, compensation and calibration, 2nd Chemnitz Parallel Kinematics Seminar, 73-83

13. Wu J.-F., Zhang R., WAng R., YAo Y., 2014, A systematic optimization approach for the calibration of parallel kinematics machine tools by a laser tracker, International Journal of Machine Tools and Manufacture, 86, 1-11 